\title{
Effects of combined training vs aerobic training on cognitive functions in COPD: a randomized controlled trial
}

This article was published in the following Dove Press journal:

International Journal of COPD

4 April 2016

Number of times this article has been viewed

\author{
Giovanna Aquino' \\ Enzo Iuliano' \\ Alessandra di Cagno ${ }^{2}$ \\ Angela Vardaro 3 \\ Giovanni Fiorilli' \\ Stefano Moffa' \\ Alfonso Di Costanzo' \\ Giuseppe De Simone ${ }^{3}$ \\ Giuseppe Calcagno' \\ 'Department of Medicine and \\ Health Sciences "Vincenzo Tiberio", \\ University of Molise, Campobasso, \\ ${ }^{2}$ Department of Health Science, \\ University Foroltalico, Rome, " "Villa \\ Margherita", Benevento, Italy
}

Aim: The aim of this study was to investigate the effects of high-intensity aerobic training (AT) and high-intensity aerobic training combined with resistance training (ie, combined training $[\mathrm{CT}])$ on cognitive function in patients with COPD.

Methods: Twenty-eight Caucasian male patients (68.35 \pm 9.64 years; mean $\pm \mathrm{SD})$ with COPD were recruited and randomized into two groups, AT and CT. Both groups performed physical reconditioning for 4 weeks, with a frequency of five training sessions per week. The CT group completed two daily sessions of 30 minutes: one aerobic session and one strength session, respectively; The AT group performed two 30-minute aerobic endurance exercise sessions on treadmill. Physical and cognitive function tests were performed before and after the training intervention performances.

Results: Exercise training improved the following cognitive functions: long-term memory, verbal fluency, attentional capacity, apraxia, and reasoning skills $(P<0.01)$. Moreover, the improvements in the CT group were significantly greater than those in the AT group in longterm memory, apraxia, and reasoning skills $(P<0.05)$.

Conclusion: CT may be a possible strategy to prevent cognitive decline and associated comorbidities in male patients with COPD.

Keywords: physical training, cognition, resistance training, rehabilitation, respiratory disease

\section{Introduction}

COPD is a complex multicomponent disorder with significant physical, psychological, and cognitive sequels that reduce the quality of life. COPD is associated with many extrapulmonary disorders that contribute to increased morbidity and mortality. ${ }^{1} \mathrm{Cog}$ nitive decline is an associated pathology ${ }^{2}$ that affects $77 \%$ of patients with COPD. ${ }^{3}$ This disturbance is probably a consequence of the neuronal damage produced by hypoxemia. Patients with COPD have an altered cerebral perfusion due to arterial oxygen desaturation ${ }^{4}$ that may result in cognitive impairments. ${ }^{5,6}$

Cognitive impairment associated with COPD differs from Alzheimer disease., ${ }^{7,8}$ The neuropsychological impairment associated with COPD has a specific pattern characterized by moderate-to-severe deficits in isolated domains, such as attention, memory, executive functions, language skill, psychomotor speed, and complex visualmotor processes. ${ }^{9}$ Exercise training is associated with maintenance and improvement of physical and cognitive functioning and psychological well-being. ${ }^{10,11}$

A meta-analysis of randomized controlled trials showed that a $14 \%$ improvement in aerobic activity coincided with an improvement in cognitive capacity. ${ }^{12}$ However,
Correspondence: Giovanna Aquino Department of Medicine and Health Sciences “Vincenzo Tiberio", University of Molise, 86100 Campobasso, Italy Tel +39874404899

Fax +39874404763

Email giovanna.aquino@unimol.it
International Journal of COPD 2016:1/ 7||-7|8

(c) (1) (8) ๑ 2016 Aquino et al. This work is published and licensed by Dove Medical Press Limited. The full terms of this license are available at https://www.dovepress.com/terms.php

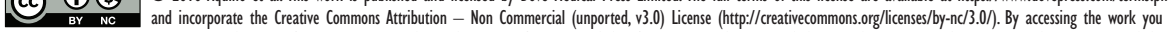
hereby accept the Terms. Non-commercial uses of the work are permitted without any further permission from Dove Medical Press Limited, provided the work is properly attributed. For peminsion for commercial use of this work, please see paragraphs 4.2 and 5 of our Terms (https://www.dovepress.com/terms.php.

\section{1}

Dovepress

http://dx.doi.org/10.2147/COPD.S96663 
despite the well-established link between exercise training and improved cognitive function, no specific training protocols exist. ${ }^{13}$ Furthermore, it is unclear if the positive effects of physical activity on cognition in patients with COPD confer long-term benefits. The majority of exercise training interventions on cognitive function have primarily focused on aerobic endurance training, called aerobic training (AT). Consequently, the aim of this study was to compare the effects of high-intensity resistance training combined with AT (ie, combined training [CT]) vs aerobic endurance exercise intervention alone on cognitive function in patients with COPD.

\section{Materials and methods}

This study is a randomized controlled study, with two measurement periods (baseline and trial completed). The assessors of the cognitive and physical tests were formally blinded, whereas the participants and the trainers were not blinded.

Twenty-eight Caucasian male patients with mildto-moderate COPD (I and II levels following the Gold Classification) ${ }^{14}$ were recruited for this study (mean age $68.35 \pm 9.64$ years). The inclusion criteria for the enrollment were as follows: age $>50$ years; former smokers, Tiffenau index (forced expiratory volume in the first second $\left[\mathrm{FEV}_{1}\right]$ / forced vital capacity [FVC] $<70 \%$ and $\mathrm{FEV}_{1}$ postbronchodilator $<80 \%$ of predicted value, reversibility of $\mathrm{FEV}_{1}<12 \%$ of basic value and $<200 \mathrm{~mL}$ of absolute value (30 minutes after $400 \mathrm{mg}$ salbutamol inhalation), and stable COPD diagnosis. The exclusion criteria were as follows: contraindication for physical activity practice; usage of oxygen therapy; evidence of dementia, evaluated by Mini-Mental State Evaluation; ${ }^{15}$ history of brain injury; history of stroke; history of alcoholism; presence of anxiety and depressive symptoms, evaluated, respectively, by Hamilton Rating Scale for Anxiety ${ }^{16,17}$ and Beck Depression Inventory; ${ }^{18,19}$ usage of medication influencing cognition; and presence of comorbidity incompatible with the experimental protocol practice. A written consent was obtained from the participants after their being thoroughly informed of the purpose and potential risks of the study. The experimental procedures were designed and conducted following the ethical principles laid out in the 2008 revision of the Declaration of Helsinki, and they were approved by the Ethical Committee of the University of Molise.

The recruitment process was conducted as follows: 72 patients with COPD at the nursing home "Villa Margherita" were enrolled during the 2 months prior to the beginning of the interventional protocols (July and August, 2014) and were considered as eligible. All these patients had not yet been included in any standard rehabilitation program. Successively, the patients were filtered on the basis of the inclusion and exclusion criteria and motivation to participate in the study. Twenty-eight participants met all these criteria. These participants carried out the physical interventions from September 1 to September 26, 2014, and they performed both the pre- and post-assessments. During the interventions, no dropouts occurred. More details about the recruitment and intervention timeline are reported in Figure 1.

The patients were randomized into two groups of equal numerosity (characteristics are shown in Table 1): the first group, called CT $(\mathrm{n}=14)$, performed a training protocol composed by high-intensity aerobic and resistance exercises, associated with respiratory, balance, and mobility exercises; and the second group, called AT ( $\mathrm{n}=14)$, performed a training protocol composed by high-intensity aerobic exercises, associated with respiratory, balance, and mobility exercises.

Both groups performed two training sessions per day, the first one in the morning and the second one in the afternoon, for 5 days/wk, for a total of ten training sessions per week, for 4 weeks. The duration of each session was 30 minutes. Before and after the 30 minutes, the patients performed 5 minutes of warm-up (walking on the treadmill at 35\% $\mathrm{VO}_{2} \max$ ) and cooldown (stretching exercises), respectively. The description of the exercises and their progression of two protocols are reported in Table 2 .

The patients in both groups were tested, before and after the intervention period, on their cognitive performance with a battery of neurocognitive tests. These tests were chosen for their high reliability and reproducibility and because they have been already used in COPD population. ${ }^{20}$

The tests are described as follows, starting from those whose results were considered as primary outcomes:

Rey 15-item memory test (or Rey test) is used to assess verbal memory. ${ }^{21}$ In this test, the examiner reads 15 words, and the patients are told to remember all the 15 presented words, independently from their order. The 15 words are read again, and the patients are required to say all the words that they remember for the second time. This sequence of listening and repeating is performed five times, and one point is assigned for each remembered word during each sequence, for a maximum score of 75 points (Rey-immediate recall). After the last sequence, the patients are distracted for $15 \mathrm{~min}$ utes, and then they are required to say all the words that they remember in order to evaluate the long-term memory performance. One point is assigned for each remembered word for a maximum of 15 points (Rey-delayed recall [DR]).

Drawing copy test is used to evaluate praxis abilities. ${ }^{22}$ In the first part of the test (drawing test I), the patient must 


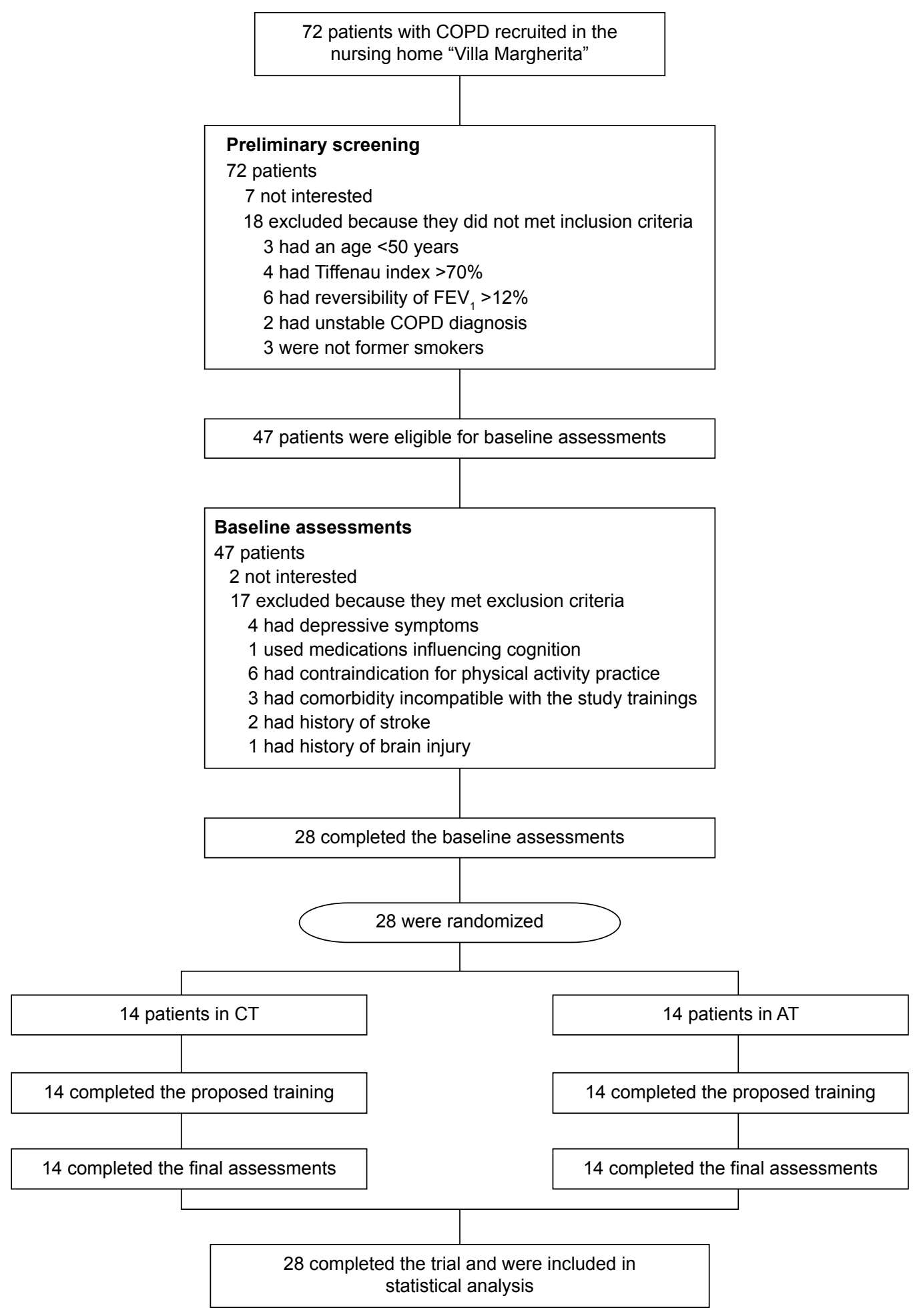

Figure I Flow-chart of the study.

Note: Combined training is aerobic and resistence training.

Abbreviations: AT, aerobic training; $\mathrm{CT}$, combined training; $\mathrm{FEV}_{1}$, forced expiratory volume in the first second.

copy three geometric drawings. The score is calculated on the basis of the adherence to the original model. The range of the assigned score is from 0 (the worst - no adherence at all) to 4 (the best - perfect adherence) for each drawing (total test score ranges from 0 , the worst, to 12 - the best). In the second part of the test (drawing test II), the patient must complete a series of 12 uncompleted geometric drawings to obtain one of the three geometric models used in the first part of the test. The score is calculated assigning one point for each correct line drawn (maximum score of 70 ), so the total 
Table I Sample characteristics

\begin{tabular}{|c|c|c|c|}
\hline & \multicolumn{3}{|l|}{ Mean \pm SD } \\
\hline & $\begin{array}{l}\text { Both groups } \\
(n=28)\end{array}$ & $\begin{array}{l}\text { CT group } \\
(n=14)\end{array}$ & $\begin{array}{l}\text { AT group } \\
(n=\mid 4)\end{array}$ \\
\hline Age (years) & $67.21 \pm 7.87$ & $65.00 \pm 8.26$ & $69.42 \pm 7.39$ \\
\hline Instruction level (years) & $9.21 \pm 5.13$ & $9.85 \pm 2.96$ & $8.57 \pm 6.87$ \\
\hline $\mathrm{FEV}_{1} \%$ & $68.42 \pm 11.54$ & $67.71 \pm 11.77$ & $69.14 \pm 10.38$ \\
\hline $\mathrm{FEV}_{1} / \mathrm{FVC} \%$ & $62.07 \pm 8.16$ & $63.28 \pm 6.96$ & $60.85 \pm 9.61$ \\
\hline \multirow[t]{2}{*}{ MRC score } & $2.70 \pm 0.95$ & $2.57 \pm 0.97$ & $2.85 \pm 0.69$ \\
\hline & \multicolumn{3}{|c|}{ Number of patients (\%) } \\
\hline \multicolumn{4}{|l|}{ Comorbidities } \\
\hline Hypertension & $26(92)$ & $13(92)$ & $13(92)$ \\
\hline Diabetes & $2(7)$ & I (7) & I (7) \\
\hline Obesity & $3(10)$ & I (7) & $2(14)$ \\
\hline \multicolumn{4}{|l|}{ Medication } \\
\hline Diuretics & $13(46)$ & $7(50)$ & $6(43)$ \\
\hline Antihypertensive & $12(42)$ & $7(50)$ & $5(36)$ \\
\hline Insulin therapy & I (3) & I (7) & $0(0)$ \\
\hline
\end{tabular}

Note: Combined training is aerobic and resistence training.

Abbreviations: AT, aerobic training; CT, combined training; $\mathrm{FEV}_{1}$, forced expiratory volume in the first second; FVC, forced vital capacity; MRC, Medical Research Council Scale.

score is from 0 (worst) to 70 (best). In this case also, the time to complete the entire test was considered as scores.

The secondary outcomes of the study were instead the results of the following tests:
Attentive matrices test (attentive test) is a valid instrument to measure the selective and sustained attention. ${ }^{23}$ It consists of three numeric matrices (ten columns of 13 numbers from 0 to 9 ). The participants are required to check specific target numbers in 45 seconds for each matrix. There is one target in the first matrix, two in the second one, and three in the third one. The score is calculated by assigning one point for each target correctly found in the three matrices (maximum of 60 for all the three matrices). The time to complete the three matrices is considered as score.

Raven's progressive matrices tests (or Raven test) are multiple choice intelligence tests of abstract reasoning. ${ }^{24}$ The test is composed of three series of 12 figures for a total of 36 figures. In each figure, the patient is asked to analyze a geometric pattern and to identify the correct missing piece among the four or six proposed pieces. One point is assigned for each missing piece that the patient correctly pointed out, so that the total score ranges from 0 (worst) to 36 (best).

Verbal fluency test is a test for the verbal fluency assessment. ${ }^{25}$ In this test, the patient is required to list, in 2 minutes, all the words he knows concerning the four categories: colors, animals, fruits and names of cities (eg, in

Table 2 Description of two protocols of training

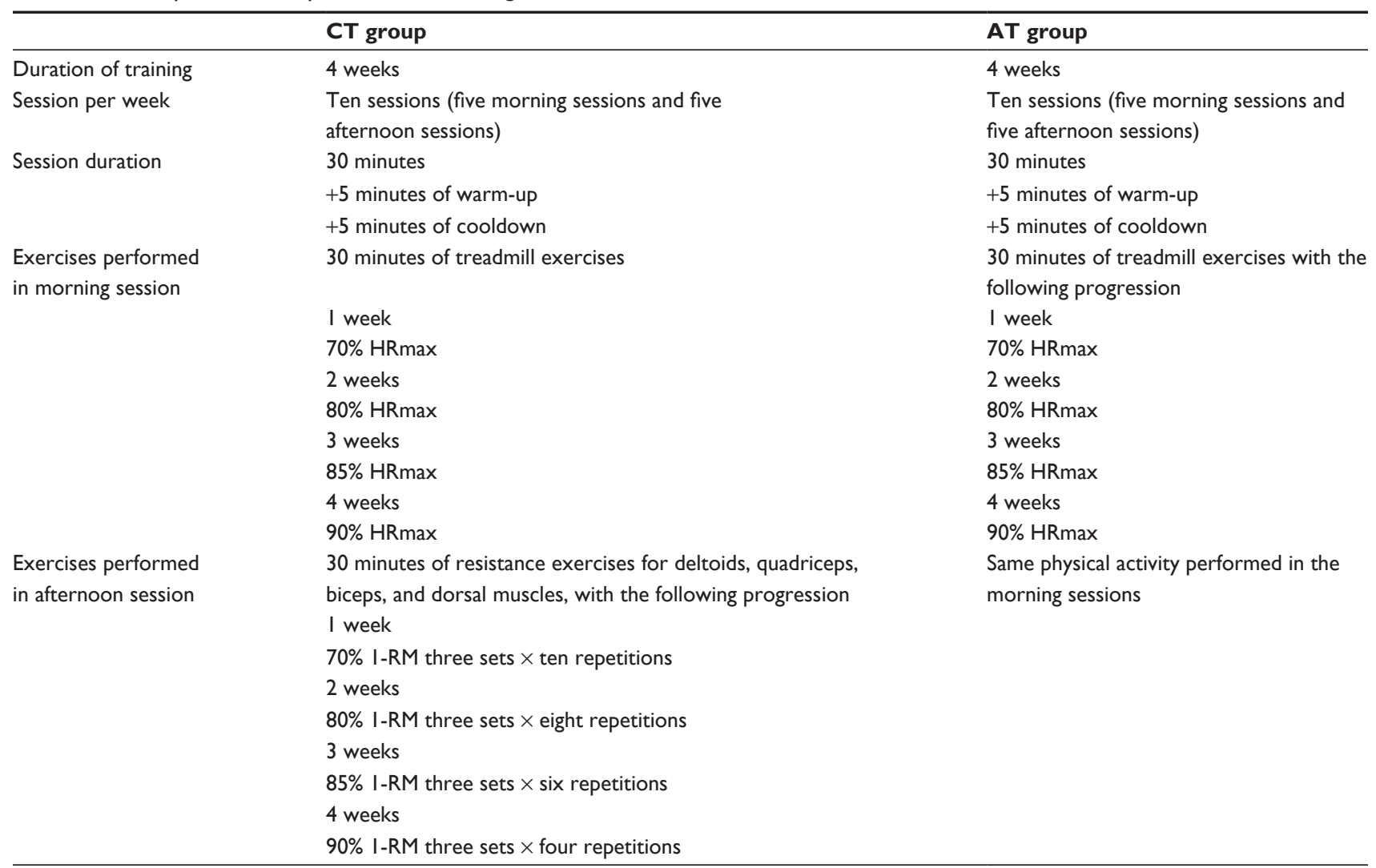

Note: Combined training is aerobic and resistence training.

Abbreviations: I-RM, one-repetition maximum; AT, aerobic training; CT, combined training; HRmax, heart rate maximum. 
the category colors, the patient has to list white, black, red, etc.). The scores are calculated assigning one point for each correct word cited, divided into four. The range of scores is from 0 (very poor) to infinite (higher score $=$ higher performance). Furthermore, the patients were tested relative to their physical levels. In order to verify the effectiveness of the proposed physical training, the patients were tested with the 6 minutes walking test, for the indirect evaluation of aerobic capacity $\left(\mathrm{VO}_{2} \max \right)$, whereas the one-repetition maximum was used to evaluate the muscle strength of quadriceps and arms (respectively, measured by leg extension and arm curl isotonic machines), and the data are reported in Table 3. The one-repetition maximum was estimated by Brzycki $^{26}$ using the submaximum method in order to assure the safety of the participants. The intensity of the aerobic sessions, expressed as percentage of heart rate maximum, was monitored in both groups through heart rate monitors. The heart rate maximum was calculated using the Tanaka formula. The Borg Scale was also used to evaluate the rate of perceived exertion.

\section{Statistics}

Participants' randomization into the two groups was performed using a random number list, generated using the online software (https://www.random.org/sequences/, Dublin, Ireland). The procedure described was as follows: a progressive number was assigned to each of the participants in alphabetical order according to their surname; a random number list was subsequently generated; and, in accordance with this random number list order, the participants were allocated in blocks of two participants per group in the order CT and AT. After randomization, analysis of variance (ANOVA) and chi-squared analysis were performed to verify that the four groups were homogeneous at baseline in terms of participants' age, instruction levels, functional status, Medical Research Council Scale scores, severity of the COPD, comorbidities, medications, and cognitive scores.

Table 3 Physical assessment results

\begin{tabular}{|c|c|c|c|c|}
\hline & \multicolumn{2}{|c|}{ CT group, mean \pm SD } & \multicolumn{2}{|c|}{ AT group, mean \pm SD } \\
\hline & Pre & Post & Pre & Post \\
\hline \multicolumn{5}{|c|}{ Aerobic capacity } \\
\hline $\begin{array}{l}\mathrm{VO}_{2} \max \\
(\mathrm{mL} / \mathrm{kg} / \mathrm{min})\end{array}$ & $7.48 \pm 2.79$ & $12.26 \pm 3.20$ & $7.63 \pm 3.00$ & $12.64 \pm 2.25$ \\
\hline \multicolumn{5}{|c|}{ Muscle strength (kg) } \\
\hline Quadriceps & $25.57 \pm 8.94$ & $37.29 \pm 13.34$ & $26.29 \pm 8.11$ & $37.36 \pm|3.2|$ \\
\hline Arms & $8.43 \pm 2.47$ & $12.86 \pm 3.25$ & $8.29 \pm 2.40$ & $11.14 \pm 2.77$ \\
\hline
\end{tabular}

Note: Combined training is aerobic and resistence training.

Abbreviations: AT, aerobic training; $\mathrm{CT}$, combined training; $\mathrm{VO}_{2}$ max, maximal oxygen consumption.
A multivariate ANOVA with repeated measures was performed to evaluate significant differences between the scores obtained in cognitive test: the two groups were used as between factor for analysis (CT vs AT), whereas the score obtained after and before the training period was used as within factor (pre vs post) and the scores obtained in the cognitive test were used as dependent variables. ANOVA with repeated measures was instead performed to evaluate significant differences on $\mathrm{FEV}_{1}$ and $\mathrm{FEV}_{1} / \mathrm{FVC}$ between the two groups and between pre- and post-evaluation.

Furthermore, Pearson correlation analysis was performed between the variations in cognitive scores and the variations in physical scores (variations calculated as $\Delta$ that was postscores minus prescores), in order to verify if a correlation existed between physical performance and cognitive modifications.

The alpha level for the analyses was set at 0.05 . The analysis was performed using the Statistical Package for the Social Sciences (v.20.0; IBM Corporation, Armonk, NY, USA).

\section{Results}

After the randomization, the two groups were homogeneous in terms of age, instruction levels, functional status, Medical Research Council Scale scores, severity of the COPD, comorbidities, medications, and cognitive scores.

The multivariate ANOVA with repeated measures showed significant differences both between the pre- vs post-exercise training scores $\left(F_{7,20}=80.884 ; P<0.001\right)$ and between the two intervention groups $\left(F_{7,20}=3.216 ; P=0.019\right)$ and in the interaction time $\times$ groups $\left(F_{7,20}=4.534 ; P=0.004\right)$. The univariate analysis showed significant differences in between pre- vs postscores in Rey-DR, verbal fluency, attentive matrices, copy drawing II, and Raven test (with $F_{1,26}$ between 27.994 and 75.214; $P<0.001$ for all the five tests). The CT group significantly improved in Rey-DR, copy drawing II, and Raven matrices $\left(F_{1,26}\right.$ between 4.872 and 5.314; $P<0.05$ for all the three tests) to a greater extent than the AT group. The univariate analysis also showed significant differences in the interaction time $\times$ groups in Rey-DR $\left(F_{1,26}=5.652 ; P=0.025\right)$, Raven test $\left(F_{1,26}=5.792 ; P=0.023\right)$, and copy drawing $\mathrm{I}\left(F_{1,26}=10.156\right.$; $P=0.004)$. These results are shown in Table 4. No significant differences were found in $\mathrm{FEV}_{1}$ and $\mathrm{FEV}_{1} / \mathrm{FVC}$ both between pre- and postevaluation and between the two groups. Furthermore, low or no significant correlations between functional and cognitive improvements were found.

\section{Discussion}

The main finding of this study concerns the improvement in cognitive function observed after 4 weeks of exercise training 
Table 4 Results obtained by the two groups in the cognitive tests

\begin{tabular}{|c|c|c|c|}
\hline & $\begin{array}{l}\text { Both groups, } \\
\text { mean } \pm \text { SD }\end{array}$ & $\begin{array}{l}C T \text { group, } \\
\text { mean } \pm S D\end{array}$ & $\begin{array}{l}\text { AT group, } \\
\text { mean } \pm \text { SD }\end{array}$ \\
\hline \multicolumn{4}{|c|}{ Attentive matrices } \\
\hline Pre & $61.93 \pm 7.2$ & $62.14 \pm 9.4$ & $61.7 I \pm 4.36$ \\
\hline Post & $64.7 \mathrm{I} \pm 6.77^{*}$ & $64.57 \pm 8.32 *$ & $64.86 \pm 5 . I^{*}$ \\
\hline \multicolumn{4}{|l|}{ Rey-IR } \\
\hline Pre & $39 \pm 9.5$ & $41.86 \pm 10.46$ & $36.14 \pm 7.77$ \\
\hline Post & $38.86 \pm 8.78$ & $41.57 \pm 9.03$ & $36 .|4 \pm 7.9|$ \\
\hline \multicolumn{4}{|l|}{ Rey-DR } \\
\hline Pre & $7.64 \pm 2.36$ & $8.29 \pm 2.7$ & $7 \pm 1.84$ \\
\hline Post & $8.64 \pm 2.57^{*}$ & $9.64 \pm 2.76^{\text {*\#\# }}$ & $7.64 \pm 1.98 * *$ \\
\hline \multicolumn{4}{|c|}{ Raven test } \\
\hline Pre & $26.79 \pm 4.65$ & $27.7 I \pm 4.6$ & $25.86 \pm 4.69$ \\
\hline Post & $27.96 \pm 4.08 *$ & $29.43 \pm 3.37^{*, \#}$ & $26.5 \pm 4.3 I^{* *}$ \\
\hline \multicolumn{4}{|c|}{ Verbal fluency } \\
\hline Pre & $34.79 \pm 11.82$ & $35.7 I \pm 13.53$ & $33.86 \pm 10.26$ \\
\hline Post & $39.29 \pm 12.68 *$ & $40.7 I \pm 14.65^{*}$ & $37.86 \pm 10.72 *$ \\
\hline \multicolumn{4}{|c|}{ Drawing test I } \\
\hline Pre & $5.86 \pm 1.08$ & $5.86 \pm|.5|$ & $5.86 \pm 0.36$ \\
\hline Post & $6.07 \pm 1.3$ & $6.43 \pm 1.55$ & $5.7 I \pm 0.9 I$ \\
\hline \multicolumn{4}{|c|}{ Drawing test II } \\
\hline Pre & $48.36 \pm 11.53$ & $47.36 \pm 11.28$ & $49.36 \pm 12.12$ \\
\hline Post & $52.82 \pm 9.27 *$ & $53.07 \pm 9.29$ *\# & $52.57 \pm 9.59 * *$ \\
\hline
\end{tabular}

Notes: ${ }^{* P}<0.01$ and ${ }^{* * P}<0.05$ in the comparison of pre vs post. ${ }^{*}<<0.05$ in the comparison of $\mathrm{CT}$ group vs AT group. Combined training is aerobic and resistence training.

Abbreviations: AT, aerobic training; CT, combined training; DR, delayed recall; $\mathbb{R}$, immediate recall.

at high intensity. This result supports the idea that physical training has the potential to improve cognitive ability in patients with COPD. ${ }^{10,27}$

The second significant finding refers to the comparison between two types of training intervention at high intensity. The CT group displayed a significantly greater improvement in the Rey-DR, Raven test, and copy drawing I assessments compared to AT group. These results suggest that the strength training combined with AT can maximize the effects of physical training on cognition in patients with COPD. There is a dearth of data investigating the effects of resistance or combined resistance and aerobic exercise training interventions on cognitive function in patients with COPD.

The majority of previous training intervention studies in cognitive functions have utilized aerobic endurance exercise in isolation ${ }^{13,28}$ performed with moderate intensity. ${ }^{29}$ The effects of physical exercise on cognitive performance appear to be attributable to various factors. ${ }^{30}$

It is well known that the physical activity influences endocrine homeostasis, such as cortisol, testosterone, growth hormone, and hypothalamic hormones, ${ }^{31}$ and the hormonal adaptation may be a possible mechanism mediating the positive effects of training. Furthermore, several hormones, influenced by physical activity, produce effects on psychological well-being through the reduction of cortisol, which causes an antidepressive effect..$^{32}$ The mood state improvement may indirectly facilitate the cognitive functions. ${ }^{33}$ Consequently, it was hypothesized that the effects of exercise on cognition may be attributable to an emotional response that introduces noradrenalin into the central nervous system (CNS) directly. ${ }^{34}$ In particularly serotonin and $\beta$-endorphin, ${ }^{35}$ give positive effects on mood and they may act as a physiological modulators for memory. ${ }^{36}$

The execution of free weight resistance exercises promotes a better development of coordinative ability, due to the higher levels of mental effort required for the executions of this type of exercises. ${ }^{37}$ Little is known about the neuronal mechanisms involved in the increased neuronal drive in the early stages of strength training, although it has been suggested that this training increased cortical drive to the spinal motoneurons. ${ }^{38}$ Strength increments arise as a consequence of numerous factors, but it would make sense to consider strength training as a kind of motor-learning process. ${ }^{39}$ Moreover, as shown in a recent study, resistance training can improve the respiratory muscle performance with potential positive effects on blood oxygenation, and consequently, the cognition performance of patients with COPD. ${ }^{40}$ Despite there being no direct evidences of the effectiveness of resistance training in improving the quality of life of patients with COPD, a recent meta-analysis ${ }^{41}$ showed that resistance training should be incorporated in COPD rehabilitation programs, for positive effects of muscle strength, especially in leg muscles. This aspect may play an important role in delaying the disease progression and also in improving patients' (including COPD) self-efficacy perception. Further studies will confirm this hypothesis. The aerobic component of the training, conducted intensively, may increase the ability of the heart to delivery oxygen ${ }^{42}$ and it is possible to assume that it could be the physiological mediator of several mental health benefits related to a better oxygenation and a greater blood flow in cerebral areas. These physiological adaptations probably compensate the hypoxemic damage that characterizes the patients with COPD. ${ }^{28,30,43}$ On the basis of previous studies, it was hypothesized that aerobic exercises may produce an increase in hippocampus volume and the stimulation of brain-derived neurotrophic factor, which positively influences the memory performances. ${ }^{28,44}$

In the human brain, aerobic exercise can increase the oxidative capability, developing a trophic effect in cerebral centers involved in sensory-motor function and in the central executive function related to the frontal lobe. 
The hippocampus volume might also be improved by high level of fitness training in humans. ${ }^{45}$

The possible beneficial effects of exercise on blood circulation in patients with COPD are also suggested by the results of a recent study of Zambom-Ferraresi et al, ${ }^{46}$ reporting a heart rate and blood lactate reduction, especially during aerobic combined with resistance training, in which strength improvements due to resistance training maximize the AT benefits. In addition, acute physical exercise, based on the combination of AT and resistance training, may improve cognitive performance by changing the levels of neurotransmitters in CNS, such as acetylcholine, dopamine, norepinephrine, epinephrine, adrenocorticotropic hormone, and vasopressin. ${ }^{47,48}$ These molecules are important neurotransmitters of the CNS and are able to promote cognitive function. ${ }^{36}$

In conclusion, this study has demonstrated from a clinical point of view that the use of combined protocol could be more useful than AT alone in order to improve cognition. These results have been recently highlighted by a meta-analysis of Kelly et al. ${ }^{49}$

A recent study concerning the exercise effects on cognition showed different benefits induced by different kinds of physical activity. ${ }^{50}$ Variety in the exercise proposals may be recommended to promote cognition improvements in patients with COPD.

\section{Conclusion}

Strategies to improve neurocognitive functioning have important health implications to contrast neural degeneration in COPD. Subclinical neurocognitive deficits are associated with an increased risk of neurocognitive impairment, dementia, and mortality. ${ }^{51}$

One strategy to improve neurocognitive functioning, which has gained increased attention, is the use of a combined exercise training intervention, in contrast to aerobic endurance training per se. For this reason, the CT protocol could be a possible means for the cognitive rehabilitation of patients with COPD. The moderate number of patients recruited may be a limitation for this study. It was due to the difficulty in recruiting patients with COPD who could be included in the experimentation. Moreover, this study did not analyze specifically the mechanism by which physical activity may improve the status of patients with COPD, and for this reason, the authors can only speculate on these mechanisms to explain the obtained findings. ${ }^{30}$

\section{Acknowledgment}

The authors wish to thank Dr Stefano Moffa for his help.

\section{Disclosure}

The authors report no conflicts of interest in this work.

\section{References}

1. Wouters EF, Creutzberg EC, Schols AM. Systemic effects in COPD. Chest. 2002;121:127-130.

2. Meek PM, Lareau SC, Anderson D. Memory for symptoms in COPD patients: how accurate are their reports? Eur Respir J. 2001;18(3): 474- 481.

3. Grant I, Heaton RK, McSweeny AJ, Adams KM, Timms RM. Neuropsychologic findings in hypoxemic chronic obstructive pulmonary disease. Arch Intern Med. 1982;142:1470-1476.

4. Ortapamuk H, Naldoken S. Brain perfusion abnormalities in chronic obstructive pulmonary disease: comparison with cognitive impairment Ann Nucl Med. 2006;20(2):99-106.

5. Dustman RE, Emmerson R, Shearer D. Physical activity, age, and cognitive neuropsychological function. J Aging Phys Act. 1994;2:143-181.

6. Marchal G, Rioux P, Petit-Taboue MC. Regional cerebral oxygen consumption, blood flow, and blood volume in healthy human aging. Arch Neurol. 1992;49:1013-1020.

7. Stuss DT, Peterkin I, Guzman DA, Guzman C, Troyer AK. Chronic obstructive pulmonary disease: effects of hypoxia on neurological and neuropsychological measures. J Clin Exp Neuropsychol. 1997;19(4):515-524.

8. Isoaho R, Puolijoki H, Huhti E, Laippala P, Kivella SL. Chronic obstructive pulmonary disease and cognitive impairment in the elderly. Int Psychogeriatr. 1996;8(1):113-125.

9. Liesker JJ, Postma DS, Beukema RJ, et al. Cognitive performance in patients with COPD. Respir Med. 2004;98(4):351-356.

10. Dodd JW, Getov SV, Jones PW. Cognitive function in COPD. Eur Respir J. 2010;35(4):913-922.

11. Kozora E, Tran ZV, Make B. Neurobehavioral improvement after brief rehabilitation in patients with chronic obstructive pulmonary disease. J Cardiopulm Rehabil. 2002;22:426-430.

12. Angevaren M, Aufdemkampe G, Verhaar HJ, Aleman A, Vanhees L. Physical activity and enhanced fitness to improve cognitive function in older people without known cognitive impairment. Cochrane Database Syst Rev. 2008;16(2):CD005381.

13. Etnier JL, Berry M. Fluid intelligence in an older COPD sample after short-or long-term exercise. Med Sci Sports Exerc. 2001;33(10): $1620-1628$.

14. Pauwels RA, Buist AS, Calverley PM, Jenkins CR, Hurd SS. Global strategy for the diagnosis, management, and prevention of chronic obstructive pulmonary disease. Am J Respir Crit Care Med. 2014; 163(5):1256-1276.

15. Peruzza S, Sergi G, Vianello A, et al. Chronic obstructive pulmonary disease (COPD) in elderly subjects: impact on functional status and quality of life. Respir Med. 2003;97(6):612-617.

16. Beck AT, Epstein N, Brown G, Steer RA. An inventory for measuring clinical anxiety: psychometric properties. J Consult Clin Psychol. 1988; 56(6):893-897.

17. Hill K, Geist R, Goldstein RS, Lacasse Y. Anxiety and depression in end-stage COPD. Eur Respir J. 2008;31(3):667-677.

18. Beck AT, Steer RA, Carbin MG. Psychometric properties of the Beck depression inventory: twenty-five years of evaluation. Clin Psychol Rev. 1988;8(1):77-100.

19. Light RW, Merrill E, Despars JA, Gordon GH, Mutalipassi LR. Prevalence of depression and anxiety in patients with COPD. Relationship to functional capacity. Chest. 1985;87(1):35-38.

20. Incalzi RA, Marra C, Giordano A, et al. Cognitive impairment in chronic obstructive pulmonary disease. J Neurol. 2003;250(3):325-332.

21. Rey A. Memorisationd'uneserie de 15 mots en 5 repetitions [storing a series of 15 words in 5 series]. In: Rey A, editor. L'examen clinique en psycologie. [Clinical examination in psychology] Paris, France: Presses Universitaires des France; 1958:139-193. French.

22. Caltagirone C, Gainotti G, Masullo C, Miceli G. Validity of some neuropsychological tests in the assessment of mental deterioration. Acta Psychiatr Scand. 1979;60(1):50-56. 
23. Spinnler H, Tognoni G. Italian Group on the Neuropsychological Study of Ageing: Italian standardization and classification of neuropsychological tests. Ital J Neurol Sci. 1987;6(8):1-120.

24. Raven J. The Raven's progressive matrices: change and stability over culture and time. Cogn Psychol. 2000;41(1):1-48.

25. Kaszniak AW, Garron DC, Fox JH, Bergen D, Huckman M. Cerebral atrophy, EEG slowing, age, education, and cognitive functioning in suspected dementia. Neurology. 1979;29(9):1273-1279.

26. Brzycki M. Strength testing-predicting a one-rep max from reps-tofatigue. J Phys Educ Recreat Dance. 1993;64(1):88-90.

27. Emery CF, Shermer RL, Hauck ER, Hsiao ET, MacIntyre NR. Cognitive and psychological outcomes of exercise in a 1-year follow-up study of patients with chronic obstructive pulmonary disease. Health Psychol. 2003;22(6):598-604.

28. Etnier J, Johnston R, Dagenbach D, Pollard RJ, Rejeski WJ, Berry M. The relationships among pulmonary function, aerobic fitness, and cognitive functioning in older COPD patients. Chest. 1999;116(4):953-960.

29. Smith PJ, Blumenthal JA, Hoffman BM, et al. Aerobic exercise and neurocognitive performance: a meta-analytic review of randomized controlled trials. Psychosom Med. 2010;72(3):239.

30. Kashihara K, Maruyama T, Murota M, Nakahara Y. Positive effects of acute and moderate physical exercise on cognitive function. $J$ Physiol Anthropol. 2009;28(4):155-164.

31. Kraemer WJ, Ratamess NA. Hormonal responses and adaptations to resistance exercise and training. Sports Med. 2005;35(4):339-361.

32. Mead GE, Morley W, Campbell P, Greig CA, McMurdo M, Lawlor DA. Exercise for depression. Cochrane Database Syst Rev. 2009;(3): CD004366.

33. Fox KR. The influence of physical activity on mental well-being. Public Health Nutr. 2009;2(3a):411-418.

34. McMorris $\mathrm{T}$, Tallon $\mathrm{M}$, Williams $\mathrm{C}$, et al. Incremental exercise, plasma concentrations of catecholamines, reaction time, and motor time during performance of a noncompatible choice response time task. Percept Mot Skills. 2003;97(2):590-604.

35. Weicker H, Strüder HK. Influence of exercise on serotonergic neuromodulation in the brain. Amino Acids. 2001;20(1):35-47.

36. Gold PE, Zornetzer SF. The mnemon and its juices: neuromodulation of memory processes. Behav Neural Biol. 1983;38(2):151-189.

37. Heyn PC, Johnsons KE, Kramer AF. Endurance and strength training outcomes on cognitively impaired and cognitively intact older adults: a meta-analysis. J Nutr Health Aging. 2008;12(6):401-409.

38. Aagaard P, Simonsen EB, Andersen JL, Magnusson P, Dyhre-Poulsen P. Neural adaptation to resistance training: changes in evoked V-wave and H-reflex responses. J Appl Physiol. 2002;92:2309-2318.

39. Jensen JL, Marstrand PC, Nielsen JB. Motor skill training and strength training are associated with different plastic changes in the central nervous system. J Appl Physiol. 2005;99(4):1558-1568.
40. Neves L, Chiappa A, Da Silva V, et al. Comparative effects of inspiratory muscle training and resistance training on respiratory and skeletal muscle strength in COPD: responses of the a pulmonary rehabilitation program. Eur Respir J. 2014;44(suppl 58):598.

41. Iepsen UW, Jørgensen KJ, Ringbæk T, Hansen H, Skrubbeltrang C, Lange $\mathrm{P}$. A combination of resistance and endurance training increases leg muscle strength in COPD: an evidence-based recommendation based on systematic review with meta-analyses. Chron Respir Dis. 2015;12(2):132-145.

42. Nelson ME, Rejeski WJ, Blair SN, et al. Physical activity and public health in older adults: recommendation from the American College of Sports Medicine and the American Heart Association. Med Sci Sports Exerc. 2007;39(8):1435-1445.

43. Baker LD, Frank LL, Foster-Schubert K, et al. Effects of aerobic exercise on mild cognitive impairment: a controlled trial. Arch Neurol. 2010;67(1):71-79.

44. Erickson KI, Prakash RS, Voss MW, et al. Aerobic fitness is associated with hippocampal volume in elderly humans. Hippocampus. 2009;19(10):1030-1039.

45. Churchill JD, Galvez R, Colcombe S, Swain RA, Kramer AF, Greenough WT. Exercise, experience and the aging brain. Neurobiol Aging. 2002;23(5):941-955.

46. Zambom-Ferraresi F, Cebollero P, Gorostiaga EM, et al. Effects of combined resistance and endurance training versus resistance training alone on strength, exercise capacity, and quality of life in patients with COPD. J Cardiopulm Rehabil Prev. 2015;35:1-8.

47. Rikli RE, Edwards DJ. Effects of a three-year exercise program on motor function and cognitive processing speed in older women. Res $Q$ Exerc Sport. 1991;62(1):61-67.

48. Poehlman ET, Gardner AW, Goran MI. Influence of endurance training on energy intake, norepinephrine kinetics, and metabolic rate in older individuals. Metabolism. 1992;41(9):941-948.

49. Kelly ME, Loughrey D, Lawlor BA, Robertson IH, Walsh C, Brennan S. The impact of exercise on the cognitive functioning of healthy older adults: a systematic review and meta-analysis. Ageing Res Rev. 2014;16:12-31.

50. Iuliano E, Di Cagno A, Aquino G, et al. Effects of different types of physical activity on the cognitive functions and attention in older people: a randomised controlled study. Exp Gerontol. 2015;70:105-110.

51. Martinez CH, Richardson CR, Han MK, Cigolle CT. Chronic obstructive pulmonary disease, cognitive impairment, and development of disability: the health and retirement study. Ann Am Thorac Soc. 2014;11(9): $1362-1370$
International Journal of COPD

\section{Publish your work in this journal}

The International Journal of COPD is an international, peer-reviewed journal of therapeutics and pharmacology focusing on concise rapid reporting of clinical studies and reviews in COPD. Special focus is given to the pathophysiological processes underlying the disease, intervention programs, patient focused education, and self management protocols.

\section{Dovepress}

This journal is indexed on PubMed Central, MedLine and CAS. The manuscript management system is completely online and includes a very quick and fair peer-review system, which is all easy to use. Visit $\mathrm{http}: / / \mathrm{www}$. dovepress.com/testimonials.php to read real quotes from published authors. 